\title{
Epidemiology and microbiology of invasive salmonellosis during a 12-year period
}

\section{$\underline{\text { M. Noni }}{ }^{1}$, K. Katsadima ${ }^{1}$, E. Kyrikou ${ }^{2}$, L. Zachariadou ${ }^{2}$, I. Kontou ${ }^{1}$, A. Michos ${ }^{1}$}

1 1st Department of Pediatrics, "Aghia Sophia" Children's Hospital, Athens, Greece

2 Department of Clinical Microbiology, "Aghia Sophia” Children's Hospital, Athens, Greece "Background" - " - - " - - " - " - - - " - - " - Salmonellosis is an infection that is a major global problem for public health. Salmonella spp. causes two clinical syndromes, non-typhoid salmonellosis and . typhoid fever. Non-typhoid salmonellosis causes gastroenteritis that usually carries on but has sometimes complications, such as bacteraemia. The aim of "the study was to investigate the epidemiology of invasive salmonella infections in blood among children aged 0-16 years in a tertiary pediatric hospital during "a 12-year period.

\section{Results}

\section{"Methods}

This is a retrospective study of children aged 0-16 years who were .hospitalized at "Aghia Sophia" Children's Hospital with invasive "salmonellosis during 2004-2016. Epidemiological, demographic and laboratory data were recorded and statistical analysis was performed. . . "

* During the 12-year study period, 73 children were hospitalized due to invasivel salmonellosis.

*The median age of the hospitalized children was 2.8 years (IQR: 1.004 .75 ). .Thirty children were girls $(41.9 \%)$ and 43 boys $(58.9 \%)$.

Forty-six children were of Greek origin (61.6\%), while 13 of them were Roma $(17.8 \%)$ and 15 were foreigners $(20.5 \%)$.

$*$ Increased incidence was observed during the summer months.

*The most frequently isolated serotypes in blood cultures were S. enteritidis, S. : group B and S. group C.

Figure 1. Incidences of invasive salmonellosis during the study period

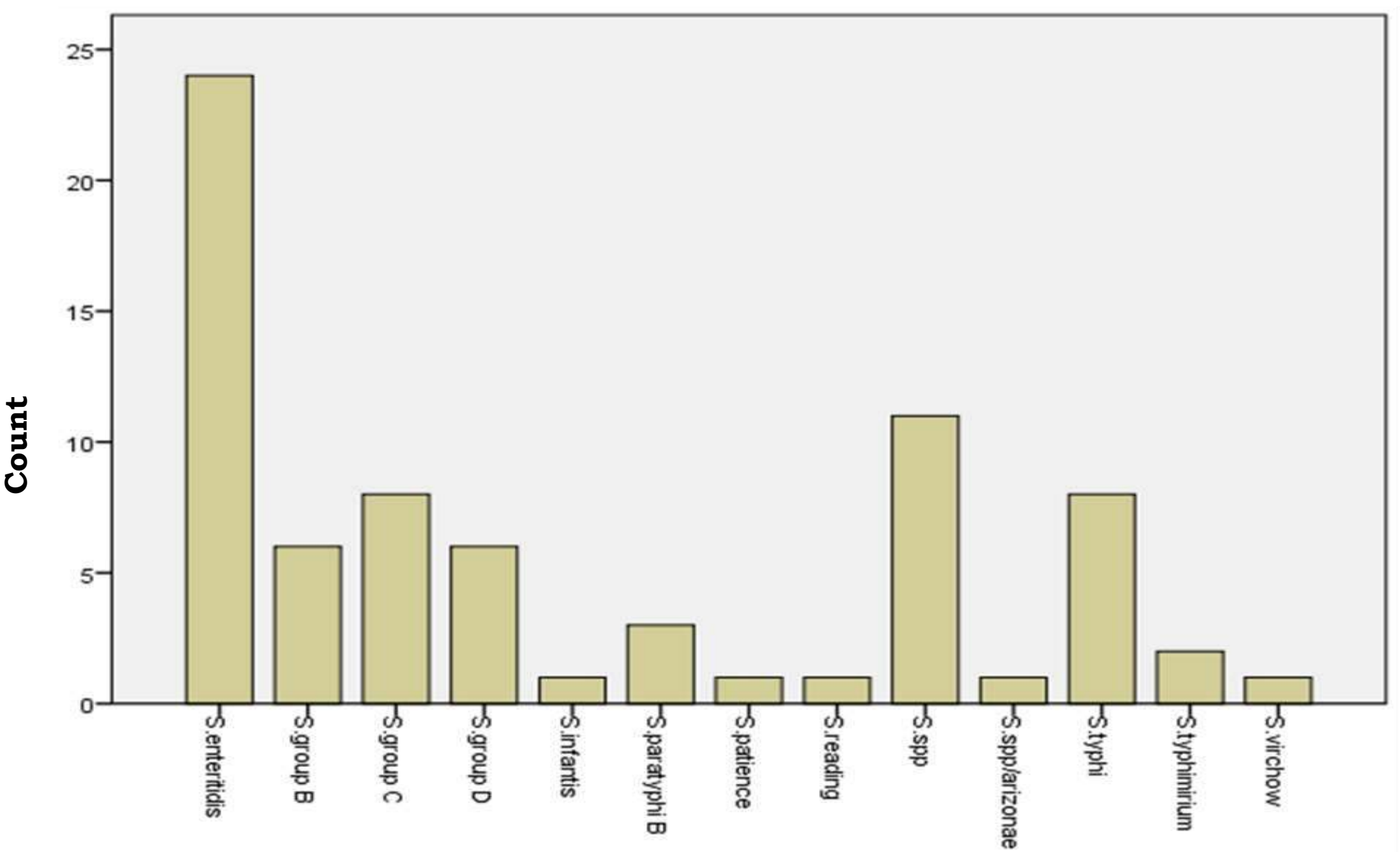

Figure 2. Distribution of Salmonella species by year

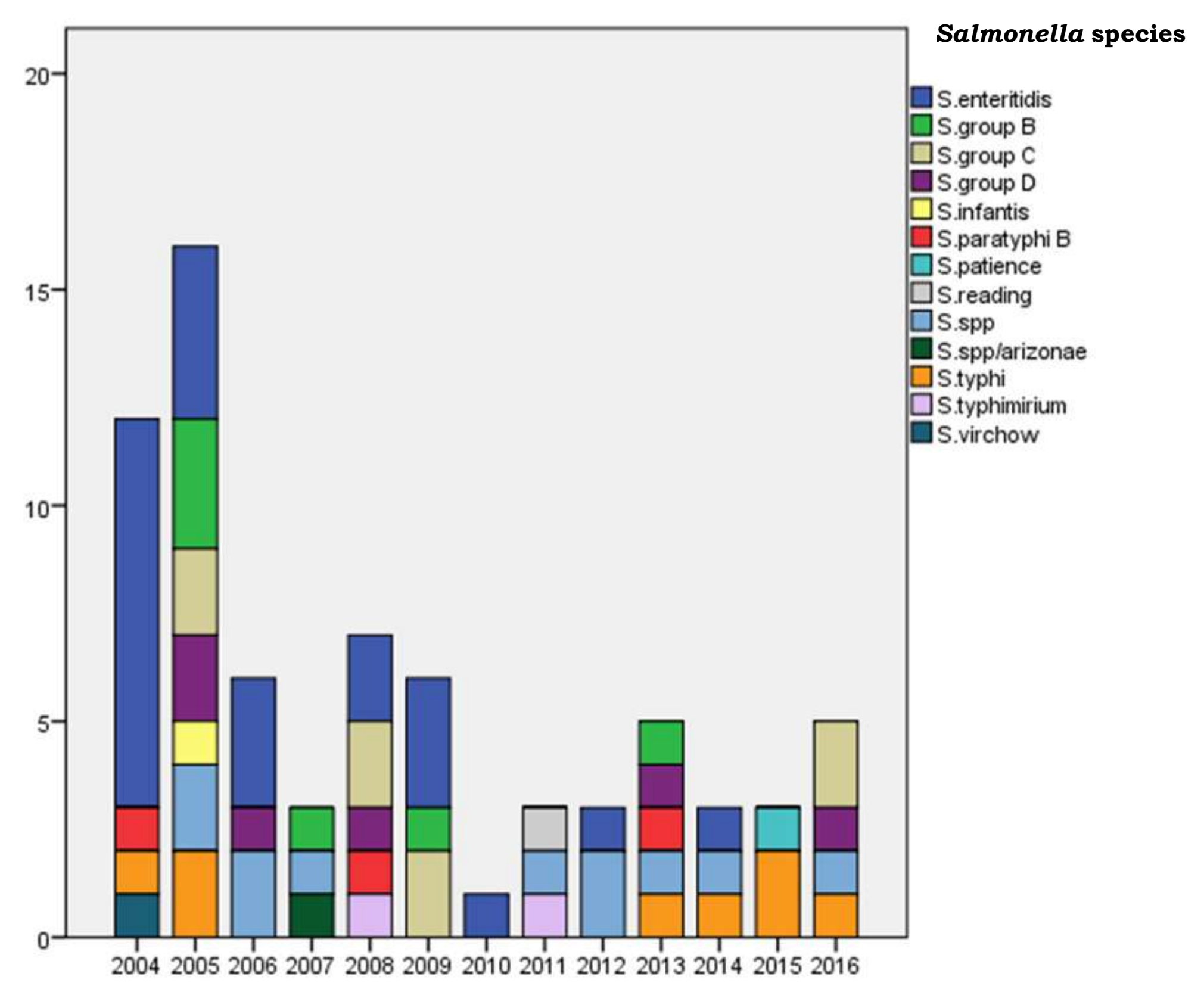

Conclusion

$\triangle$ In our study salmonella bacteremia is caused mainly by non-typhoid "serotypes, such as S.enteritidis, S.group B and S.group C, and was found to have increased incidence in infancy, especially during summer months. $>$ Further investigation of invasive Salmonella infections will substantially contribute to improving public health and preventing food-borne infections." 